\title{
An in-depth exploration of the experience and sense- making of transactional analyst psychotherapists working with clients who present with Internet addiction
}

\author{
(C) 2015 Matthew Shorrock
}

\begin{abstract}
Four internationally-accredited transactional analysis psychotherapists completed semistructured one-to-one interviews that explored their experiences and sense-making of Internet addiction (IA). Interpretive phenomenological analysis yielded four higher-order concepts: the complexity of IA; aetiological and predisposing factors; functions and features of IA; and treatment factors. Practical and theoretical implications for future research, clinical supervision, treatment, psycho-educational and political programmes are presented. Of the key emergent findings the Internet was understood by participants as a conduit or medium for addiction given a high prevalence of an underlying 'disorder'. It was also found that participants believed in the existence of childhood aetiological roots underpinning comorbidity with IA; that attachment difficulties in childhood often predispose individuals to develop issues around loneliness, low selfesteem, control, loss, instability and cognitive dissonance later in life; and that a relationship exists between depression, low self-esteem and escapism as contributing factors. It is concluded that professionals would benefit from specific trainings concerning childhood attachment difficulties, whilst integrating a psychodynamic approach, or being aware of transference processes, could enhance treatment effectiveness and help safeguard both clients and therapists from counter-therapeutic interventions.
\end{abstract}

\section{Key words}

Internet addiction, treatment, aetiology, interpretive phenomenological analysis (IPA), transactional analysis, counselling psychology.

Editor's Note: This paper presents a synopsis of a thesis submitted as part of a Professional Doctorate in Counselling Psychology; after publication of this paper, the full thesis will be available at the supplementary website associated with this journal: www. TAresearch.org. mid-August.

\section{Introduction}

My interest in the internet began when I was a teenager, progressing into an interest in Internet addiction when I became a counsellor after realising how many hours I had spent in the past 'surfing the net' in extremis. I am now clinical director of an international, community-based therapy and research institute that specialises in addictions and have noticed a dramatic increase over the past five years in the numbers of referrals concerning Internet associated problems. Hence, it seemed logical that I undertake research in this area, with a view to being able to integrate new ideas and treatment methods into my own practice and those of others.

\section{Addiction and Internet Addiction}

Young, Yue, and Ying (2011) define addictions as “... the habitual compulsion to engage in a certain activity or utilise a substance, notwithstanding the devastating consequences on the individual's physical, social, spiritual, mental, and financial wellbeing" (p.6). By developing a pseudo-coping mechanism the addict defends against facing life's challenges, daily stress and past or current trauma.

Young (1996) had adapted the DSM-IV criteria for pathological gambling to measure the (then) new phenomenon of internet addition (IA), after reviewing 600 cases of heavy Internet users. At various times since then other terms have been used: Internet Addiction Disorder [IAD] (Goldberg, 1996; Warden et al. 2004), Pathological Internet Use (Young 1996), Problematic Internet Use [PIU] (Caplan, 2002; Goyette \& Nadeau, 2008; Shapira, Goldsmith, Keck, Khosla, \& McElroy, 2000), Pathological Internet Use (Davis, 2001; Morahan-Martin \& Schumacher, 2000), Compulsive Internet Use (Greenfield 1999; 
Widyanto \& Griffiths, 2006), High Internet Dependency (Davis, Flett, \& Besser, 2002; Hur, 2006), Virtual Addiction (Greenfield, 1999), InternetEnabled Compulsive Behaviour or Digital Media Compulsion (Greenfield (2011).

At this time, there are no diagnostic instruments for Internet addiction that show adequate validity and reliability across countries. The most commonly used questionnaire is Young's Internet Addiction Test [IAT], which has been validated in the United States, Finland, Korea, as well as in the United Kingdom (Widyanto \& McMurran, 2004). Other scales include: the Chen Internet Addiction Scale [CIAS], the Questionnaire of Experiences Related to Internet [QERI], Compulsive Internet Use Scale [CIUS], and the Problematic Internet Use Questionnaire [PIUQ]. However, given that these instruments are based upon different theoretical and philosophical foundations, they do not necessarily agree on the underlying dimensions that constitute problematic Internet use (Weinstein \& Lejoyeux, 2010).

\section{Literature Review}

Despite the difficulties inherent in the endeavour, there has been a surge of research interest across the world attempting to estimate how prevalent Internet addiction is. Despite the well documented increase of individuals presenting to clinics with Internet related problems, King et al. (2011) have warned of the inclination to overestimate the severity and extent as a result of the poor methodological design within studies. Byun et al. (2009) support this claim in a metasynthesis of quantitative studies conducted between 1996 and 2006, and discovered that a large proportion of studies are flawed by a serious sampling bias, due to inconsistent identification criteria recruitment methods. However, seven years have elapsed since the cut-off point within the Byun et al. (2009) study and many more quantitative studies have since been conducted, necessitating the need for a contemporary replication of this study.

A study related to North America, which relied on self-reported data only, claimed that $6 \%$ of 17,000 respondents fitted the Internet addiction profile (Greenfield 1999). Aboujaoude et al (2006) generalised from their findings that at least one in eight in the USA experienced significant distress associated with Internet addiction. Young and Abreu (2011) reviewed a Statistical Report on Internet addictive behaviour in China (Cui, Zhao, Wu, \& Xu, 2006) that found $9.72 \%-11.06 \%$ of the 100 million internet users in China who are younger than 24 years are classified as serious addicts (an estimated 10 million young people). Using the Pathological
Internet Use (PIU) scale on students in the United Kingdom, 18.3\% were considered to be pathological Internet users (Niemz et al, 2005).

Young (1999) and Young et al. (2000) categorise Internet addiction into five subtypes: computer addiction - i.e. pathological computer game playing; cybersexual addiction - i.e. excessive use of the Internet for cybersex and cyberporn; information overload-i.e. disproportionate surfing of the Internet and searching for information; cyber-relationship addiction - i.e. an excessive engagement with virtual relationships, such as those generated through social networking sites (SNS's); and net compulsions - i.e. compulsive behaviours, such as online gambling, stock exchange dealing, and online shopping. There is some evidence that younger adults (i.e. below mid-twenties) are more vulnerable to developing internet addition than older users (Soule et al., 2003; Thatcher \& Goolam, 2005). Morahan-Martin (2005) suggests that the profile of a heavy Internet user is somebody who is experiencing loneliness, depression, or sexual compulsion. However, it would seem that there is no consensus on a stereotypical Internet addict, not to mention the profile of a user that may become a dependent user.

Greenfield (1999b, 2011) provides a helpful summary the main factors that are considered to be characteristic of the addictive potential of the Internet: content; process and access / availability factors; reinforcement and reward; and social factors. 'If content is the raw material, then the Internet medium is the psychological syringe that delivers the content into our nervous system for consumption' (Greenfield, 2011: p.140). Zeigarnik (1967) first noted that there exists an inherent tendency of the human brain to complete tasks i.e. the Zeigarnik effect. Thus, the limitless bounds of the Internet heightens an unconscious need to complete unfinished business.

The pleasurable effects derived from the use of the Internet serves as a positive reinforcer i.e. a basic tenet of operant conditioning (Ferster \& Skinner, 1957). If looking at pornographic images is the primary gain, then the consequent elevation in dopamine levels is the secondary gain, and further reinforces the addictive pattern. Social factors were identified by Greenfield (1999b, 2011) as key elements - the internet is the first medium to ever socially connect while simultaneously disconnecting its users. It enables a certain mastery in mediating the desire to connect, whilst reducing the social anxiety that can emerge in real-time off-line social 
interaction. For its users, the Internet represents a safe and predictable world.

The perspectives of cognitive-behavioural therapy (CBT) and transactional analysis (TA) represent two of the key competing yet distinct modalities attempting to understand the causal factors of Internet addiction development.

From a CBT perspective, Caplan (2002) identifies a model that highlights core components of Internet addiction (that is, salience, mood modification, tolerance, withdrawal, conflict, and relapse introduced above). In terms of empirically demonstrating the effect of a behavioural intervention, Twohig and Crosby (2010) provide some promising evidence that Acceptance Commitment Therapy (ACT) significantly reduced Internet usage by $83 \%$ within its participants. Davis (2001) has developed a cognitive-behavioural theory of pathological Internet use (PIU) that also stresses the importance of specific cognitive patterns relating to the Internet, that drive individuals into realising negative life outcomes. Using the Internet Addiction Test (IAT), the first global psychometric measure of Internet addiction, developed by Widyanto and McMurren (2004), Young (2007) evaluated Internet addiction experience as well as treatment outcome of 114 patients before, during and after the 12 session CBT intervention. 96\% of patients struggled with on-line time management, and $85 \%$ reported significant relationship problems due the amount of time spent on-line.

From a TA perspective, recent quantitative research conducted in Taiwan, using an interpersonal psychotherapy to evaluate a large sample of individuals [ $n=555]$, has attempted to identify predisposing Internet addiction factors (Liu \& Kuo, 2007). Using a self-completion questionnaire, results provided some convincing evidence that dysfunctional interpersonal relationships in childhood and consequent social anxiety significantly and positively correlate, impacting on the emergence and severity of Internet addiction. Although the results were based on a large sample ( $46 \%$ male), it is worth noting that the population targeted was inclusive only of students, and no indication was made regarding their age, and therefore indicates a methodological shortfall of this study.

Very little research has been published evidencing the value of transactional analysis (TA) in 'treating' Internet addiction, even though it provides a strong theoretical basis for explaining its origin. Shorrock (2012a, 2012b, 2012c, 2013) supports claims made by Liu and Kuo (2007) that primal dysfunctional interpersonal relationships and consequent social anxiety are key indicators in predicting the emergence and severity of Internet addiction. TA can be considered as a philosophical and conceptual integration of psychodynamic and CBT theory, and Shorrock (2012b) has attempted to capture the strength that TA offers in explaining the aetiology of psychological distress, including addictions, which are viewed as symptoms of contaminated beliefs developed in childhood. In an in-depth clinical case study (Shorrock, 2012a, 2012b) used a TA perspective to account for the origins of a client's distress caused by on-line pornography addiction; the therapy spanned a four year period, and it was only through working with the client's core dysfunctional beliefs, informed by primal injunctions (Goulding \& Goulding, 1976) that second-order characterological change could be affected. However, a major limitation of the case study is the lack of quantitative data to support qualitative evidence documenting the therapeutic gains made.

Shorrock (2013) conducted a systematic review into antecedents and predisposing factors which yielded eight themes:

1. Pre-existing conditions or evidence of comorbidity - For instance: depression and anxiety disorders, physical disabilities, obesity, sexual compulsivity, paraphilia.

2. Internet is a conduit - The Internet is merely a means of expressing another underlying disorder/ symptomatic of underlying problems.

3. Internet is not intrinsically addictive - Internet itself is not addictive, specific applications play a significant role in the development of pathological Internet use.

4. Intra-personal problems - Fulfils psychological deficiency needs, including: need for confidence / self-esteem; need for power / control; need for pleasure; need for sex / release of sexual tension; need for achievement; and need to 'create a persona'.

5. Interpersonal problems - Compensates for social / interpersonal difficulties, including: shyness; introversion; inhibition; loneliness; sense of belonging; social isolation / withdrawal; relationship breakdown.

6. Escapism / Coping - Internet provides means to avoid or cope with difficulties.

7. Normalisation - Internet provides a means to normalise 'deviant' or conflicting (ego-dystonic) thoughts and behaviours. 
8. Historical explanations - Unmet emotional needs in family of origin / childhood.

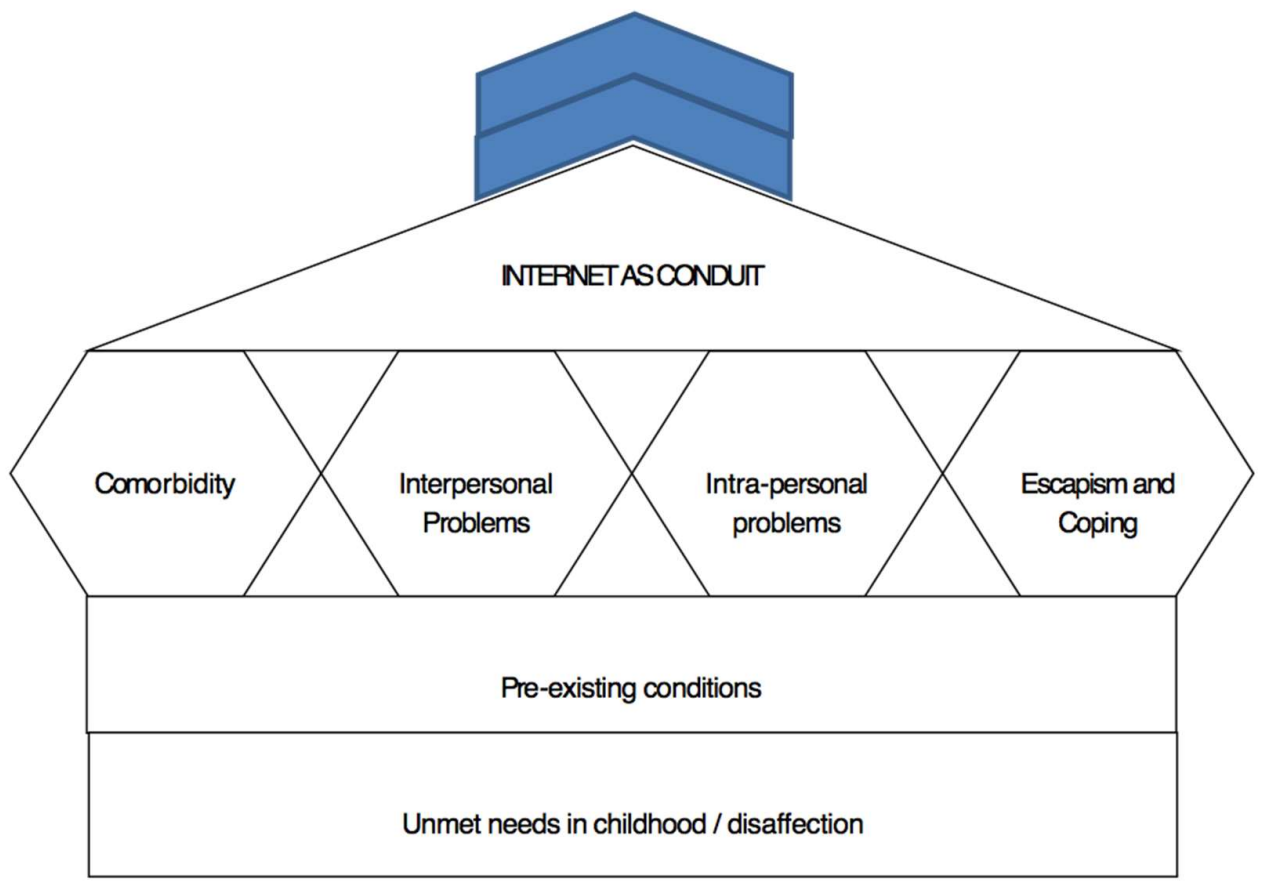

Figure 1 The Internet Addiction Aetiology Model Shorrock 2012

Shorrock noted that a third of the studies that met the inclusion criteria incorporated a research design feature that enabled the authors to conclude that unmet emotional needs in childhood heightened their subjects' vulnerability to developing Internet addiction.

He went on to present a diagrammatic conceptualisation of the emergent aetiological themes and components, as shown in Figure 1.The model can provide a 'bottom-up' understanding of the levels of complexity involved in developing an Internet addiction. Unmet needs in childhood and associated early on-set psychological conditioning can often be held to account for the development of the future addiction, (represented at the base of the model). A disaffected childhood can give rise to unhealthy psychological problems in the 'here and now' real world context (for example, relationship difficulties). The hexagonal shapes denote the interrelatedness of the contributing factors that can fixate the addiction, thus reinforcing the addictive cycle. "At this level, these factors can be understood as the everyday features and function of Internet addiction, as uniquely developed and experienced by the client. Almost like a poorly insulated roof, the addiction invariably 'leaks' out of the person, and affects their being in the world." (Shorrock, 2013, p. 13).
The main limitation of this study was the small sample size, due to the dearth of qualitative research published that investigates aetiology and predisposing factors contributing to the development of Internet addiction. The validity and reliability of the findings could be enhanced by a replication of the search strategy by a number different researchers. Finally, the findings are inclusive only of data emanating from qualitative based studies. Future research could repeat the enquiry targeting studies exclusively employing a quantitative approach, a mixed methods design, or both, to support a triangulation of data.

Treatment outcome studies are still in their infancy. King et al. (2011) conducted a comprehensive review of the research specifically focussed on clinical interventions for treating Internet addiction, using the CONSORT (Consolidating Standards of Reporting Trials) statement, an internationally recognized 'gold standard' for assessing the reporting quality of clinical trials (Altman et al., 2001). Their robust assessment of the extant clinical trials of IA treatment revealed that the majority of studies employed non-pharmacologic intervention, included CBT, motivational interviewing (MI), reality training, or a combination of psychological and/or counselling modalities within a self-directed treatment program - 
i.e. a combination of a number of therapy types. Thus, there was a lack of consistency across the reviewed studies in the type of therapy provided. King et al. (2011) highlighted several key limitations across the studies:

- inconsistencies in the definition and diagnosis of Internet addiction;

- a lack of randomization and blinding techniques;

- a lack of adequate controls or other comparison groups; and

- insufficient information concerning recruitment dates, sample characteristics, and treatment effect sizes.

Therapists' Experience \& Understanding of Working with Internet Addiction

Very little research has been conducted into therapists' experience of working with clients struggling with addictions. Shinebourne and Adams (2007) studied the conceptualisations and beliefs about addiction in research and other literature that has emerged from the USA, and conclude that they reflect a domination of the pathological / deficit model (for instance: Hshieh \& Srebalus, 1997; Humphreys et al., 1996; Moyers \& Miller, 1993; Thombs \& Osborn, 2001; Toriello \& Leierer, 2005). Shinebourne and Adams (2007) found that only one study focused on therapists' experiences, emotions, and feelings i.e. Najavits et al. (1995). It would seem the Shinebourne and Adams (2007) study still reflects current trends.

Published research undertaking an in-depth examination of the experience and understanding of therapists working with clients struggling with Internet addiction is virtually non-existent (Shorrock, 2012a; Shorrock, 2012b; Shorrock, 2012c). It is impossible to attain unadulterated access to the actual experience of a therapist; as researchers we are twice removed in attempting to comprehend the participants' narrative of their experience in working with a client (Westland \& Shinebourne, 2009). Paradoxically, this unique vantage point provides the researcher a meta-perspective enabling an identification of the therapist's 'operative defences and self-deceptions' that were present in the original clinical encounters, and can be evident in the participant's current narrative (Churchill, 2000, p. 44).

Shinebourne and Adams (2007) have attempted to explore therapists' understanding and experience of working with addiction problems generically. Participants were 13 therapists from diverse backgrounds and working settings. $A$ ' $Q$ methodology' was employed, and four distinctive factors were identified, indicating divergent understandings of the concept of addiction. Whilst a number of limitations are evident within their study, including unexplored, or partially explored data, their research prompts serious consideration of the following factors:

- Therapists' personal beliefs and values regarding addiction impact on their understanding and practice. The authors found that most of their participants' views were informed by more than one theoretical perspective.

- Therapists need to be aware of the potential impact of their values on their client work, given that clients introject their therapists' values (Berne, 1966; Kelly \& Strupp, 1992).

- Therapists' attitudes and feelings towards addictive clients indicate a need for supervision and further training. Working with clients struggling with addiction can give rise to complex situations, which in turn can elicit uncomfortable and confusing experiences for the therapist.

Most recently, spurred by the recognition that addiction counsellors perhaps possess the most advanced clinical knowledge relating to IA, Acier and Kern (2011) attempted to capture and analyse the counsellors' perceptions relating to: the profile of individuals presenting with problematic Internet use (PIU); the clinical picture; assessment; and treatment. The authors used a qualitative research design, utilising four focus groups with 21 addiction counsellors working in public addiction rehabilitation centres in Quebec, Canada. Further to an analysis of the discussions that unfolded, the authors conclude their paper with a strong note of caution, and warn us against "pathologising" a still very little known phenomenon.

A key shortcoming of this study is the undisclosed qualifications and experience of the counsellors in working with Internet addicted clients. Although the addiction counsellors have an abundance of experience working with alcohol and drug clients for a mean of 12.6 years, the only reported experience of working with clients struggling with Internet addiction is that they all they confirm they have worked with several Internet problematic use cases (mean of $6.4, \mathrm{SD}=3.62$ ) in the last 12 months only. Future research would benefit from raising the threshold, and / or clarifying inclusion criteria for 
actual experience of working with clients struggling with Internet addiction.

\section{Aims \& Rationale}

The aim of this study was to explore transactional analysis psychotherapists' experience and understanding of working with clients who present with Internet related problems.

The intention was to engage with the therapists' broader experience and understanding of therapeutic assessment, diagnosis and treatment of clients rather than the client's phenomenological account.

This issue has broad implications for social care, education and IT sectors worldwide, as the use of Internet and Internet based technologies increases exponentially. Recent figures reveal that teenagers are exposed to various technologies (including 3rd generation phones, gaming and Internet) an average of seven hours per day (Rideout, Foehr, \& Roberts, 2010). A recent and extensive literature review (Kuss \& Griffiths, 2011) revealed the negative correlates of Social Networking Site [SNS] usage to be: problematic face-to-face [FtF] interpersonal relationships; withdrawal of participation in 'real world' social communities; as well as depleting academic performance and achievement.

Husserl advocates a thorough and robust phenomenological account of the world as an essential grounding for any further scientific account. Husserl viewed science as a second-order knowledge system, ultimately depending on firstorder personal experience (Husserl, 1970). In my introduction, I have exposed the overwhelming deficit of quantitative as well as qualitative research into the Internet addiction phenomena.

\section{Methodology}

Westland and Shinebourne (2009) have employed an interpretative phenomenological analysis (IPA) methodology for investigating therapists' experience of working with clients that provides promising evidence that this approach lends itself better to a rich and in-depth exploration of therapist experience and understanding. Exploring how events and objects are experienced and how therapists makemeaning of this necessitates an interpretative activity on the part of the researcher (Smith \& Osborn, 2003; Smith et al. 2009); these are the dual foci of IPA.

Further to a systematic review (Shorrock, 2013), and two in-depth case study papers (Shorrock, 2012a; Shorrock, 2012b), methodological weaknesses within many of the studies exploring Internet addiction, include over-reliance on survey data and self-reported data from self-selected populations, and absence of control groups within quantitative research (Young \& Abreu, 2011). Previously reported case studies exploring the phenomenon (for instance, Young, 1996), have lacked depth and substance, which has recently been addressed (e.g. Chirban, 2006; Shorrock, 2012a, Shorrock, 2012b), even although studies remain scant.

Attempts have been made to explore the therapists' experience and understanding of working generically with addictions (Najavits et al, 2000; Shinebourne \& Adams, 2007). An encouraging study by Acier and Kern (2011) offered some qualitative evidence, resulting from focus groups endeavouring to capture the perceptions of addiction counsellors working with clients struggling with problematic Internet use (PIU) in Canada. However, to date, no in-depth research has been conducted, or at least published, concerning both therapists' experience and understanding of working with clients who present with Internet related problems.

The Interpretive Phenomenological Analysis (IPA) (Smith, Flower and Larkin 2009) method is a congruent qualitative approach in addressing the aims of this study. I view its three key philosophical and theoretical bedrocks as prisms:

Idiography is the study of the individual, or of single events or facts (Oxford English Dictionary Online, 2015). Commensurate with psychological therapy, idiography represents IPA's commitment to the careful examination of detail, and depth of analysis. Also, because phenomenological experience is uniquely embodied, situated and perspectival (Smith, 2011) the ideographical philosophy of IPA lends itself well toward a case study examination of a small, purposefully-selected, well placed sample of therapists who have worked closely with Internetaddicted clients.

In line with Husserl (1859-1938), phenomenology involves the setting aside of presuppositions about a phenomenon as an empirical object and about the mental acts concerned with experiencing it, in order to achieve an intuition of its pure essence and hence to analyse the structure of conscious subjective experience.

Hermeneutics has been defined as the art or science of interpretation (Ricoeur, 1981). Although historically developed to interpret text (particularly biblical scriptures), phenomenologically-minded hermeneutic researchers and psychologists can adapt the theory as a method within a face-to-face 
dialogic encounter, such as interviews. Smith and Osborne (2003) introduce the phrase double hermeneutic - the idea that the IPA researcher is attempting to make-meaning of the participant attempting to make-meaning of their experience.

Whilst I adopt the same methodological position as Smith, I cannot be as confident as to say that my account is not influenced by any of the psychological trainings I have ever received. Instead, I lean towards Heidegger (1962) and accept that it is impossible to rid the mind of presupposition and where possible, choose to work with my forestructures as an invaluable guide to a mature, meaningful, and multifaceted enquiry.

\section{Research Activity}

Subjects

Participants chosen for the study had professional membership with the European Association of Transactional Analysis (EATA) because this assured me of an integrative approach with a strong focus on examining aetiology, and a particularly high degree of rigour in levels of training, clinical competence, personal therapy, and extensive therapeutic experience necessary for their registration (ITA, 2008). Membership of EATA meant also that they would meet any national requirements for psychotherapists to practice.

To find participants, a notice was placed within a professional journal that attracted an international readership; it included clarification of the purpose of the study, along with inclusion criteria and a description of the requirements of a recorded one and a half hour semi-structured interview and contact details of the researcher. Social and professional networking media was also used to recruit participants, namely Twitter, Linkedln, and Facebook.

Responding potential participants possessed a minimum of five years practice experience, within a number of health care settings. A key requirement was that each practitioner has direct experience of working therapeutically with adult clients who have experienced problems associated with Internet addiction and compulsive Internet behaviours. Although TA is essentially a theoretically integrative approach, another pre-requisite was that therapists identify themselves as 'integrative' therapists, and have trained in or are influenced by 'other' therapeutic modalities (e.g. psychodynamic, CBT, or Rogerian person-centred therapy), even though they understand TA to be their 'home base' or core modality.
An information sheet was forwarded to potential participants, who responded with a signed copy of the research consent form. Participants that met all of the inclusion criteria, and who had provided informed content to participate, were selected on a first-come-first-serve basis.

'Participant 1' ('Jack') is an extensively experienced psychotherapist based in the UK, originally trained in second-wave CBT before his transactional analysis training. He works within the NHS and private practice, offers supervision to therapists working within internet addition, and has written academic papers on the topic.

'Participant 2' ('Dora') has specialised in using transactional analysis and other humanist approaches for over two decades, and has predominantly worked within the not-for-profit sector within the UK. Dora currently works with young single mothers who are dependent upon state benefits, or illegal income revenue, and chiefly uses a group therapy format, using both off-line and on-line models.

Participant 3' ('Kam') specialises in working with children and young people who are struggling with on-line gaming addiction. Based in Germany, Kam has established an international research and treatment institute that aims to evidence the efficacy of systemic / family based therapies using a transactional analysis modality.

'Participant 4' ('Lucy') is a highly experienced teaching and supervising transactional analyst based in the UK. She integrates narrative and attachment therapy, and has a special interest in using metaphor in her work, which she employs within a very relational and psychodynamic approach.

This sample was to represent a 'perspective' rather than a population (Smith, Flowers \& Larkin, 2009), with the added value of the standardisation of their theoretical and clinical approach due to TA being their core modality.

\section{Interviews}

All interviews were carried out at TA conference in the UK, over a period of 3 days in April 2012. Having memorised the interview schedule, I embedded deliberately open-ended questions within a conversational style of interviewing that was freeflowing and organic in nature. This enabled a rich and meaningful phenomenological enquiry to unfold. As explained previously, it was expected that the interview process would oscillate between levels of 
interpretive enquiry from a hermeneutics of empathy to a hermeneutics of suspicion (Ricoeur, 1970). The twelfth question was conceived post hoc, during the first interview. The significant value it added confirmed the decision to include it in all of the subsequent interviews, and so the questions became:

\section{Background}

How would you describe yourself as a therapist?

To what extent does TA inform your practice?

\section{Assessment}

What is your experience of working with people who have problems associated with the Internet or Internet related devices? (including smart phones and 'apps').

What does the term "Internet addiction" mean to you?

How do you evaluate whether somebody is addicted to the Internet?

What is it like for you working with people who struggle with Internet related problems?

\section{Aetiology}

Do you find that predisposing factors influence a client's expression of Internet addiction? If so, how?

What are the key factors impacting on the client's expression of Internet related problems?

Is there a link between the client's previous experience and specific types of Internet addiction?

\section{Treatment}

How does the client's previous life experience impact on your formulation of a treatment plan?

Can you walk me through your experience of working with and treating one client with Internet addiction?

\section{Reflection}

What has it been like for you today, exploring and reflecting on your understanding and experience of working with clients struggling with Internet addiction?

\section{Data Analysis}

To date, the IPA literature has not advocated one single 'method' of data analysis. However, the analytic focus has always centred on the participant's effort to understand and make-meaning of their experience. Smith (2007) has described the analytic process as an iterative and inductive cycle.

Using the final texts, I followed the sequence of data analysis below, which did not always necessarily follow a linear process, as I needed to allow myself to oscillate reflectively between stages as part of an iterative and inductive cycle of analysis (Smith, 2007):

Reading and re-reading.

Taking of initial notes, making descriptive, conceptual, linguistic comments.

Identifying emergent patterns / themes, and connections across themes.

Repeating the above process with the next participant's interview transcript.

Detecting patterns across case transcripts.

The data analysis process started from the moment the interview process began, and even before, upon making initial contact with the participant. Recognition of a 'double hermeneutic' (Smith \& Osborn, 2003) within the interview process is congruent with an IPA methodology, acknowledging that the researcher is 'both like and unlike the participant'. I made use of data from my own reflexive journal, and research supervision, to aid data analysis and interpretation of the participant's experience (Etherington, 2004). Not wishing to discount the importance of my own fore-structures (Heidegger, 1962 / 1967) and indeed utilising them in the service of a double-hermeneutic of suspicion as well as empathy (Ricoeur, 1970), I was also mindful of the psychodynamic lens of which I would be interpreting the data.

\section{Ethical Considerations}

In addition to the ethical codes I follow due to my dual professional roles (as a psychotherapist and psychologist), as a member of the British Psychological Society I also followed the BPS (2010) Code of Human Research Ethics.

The universal themes for consideration within the range of codes were confidentiality awareness, avoidance of harm to participants, and procedures for dealing with potential distress caused to participants (McLeod, 2003). However, Smith, Flowers and Larkin (2009) have noted that "anonymity is all that qualitative researchers can offer", and are at pains to stress the point that "to say that something is 'confidential' is to say that nobody else will see it" (p.53), which cannot be the case. I therefore intended to delete any names that would appear in the text, and alter any information that could remotely identify the participant or the clients to which they referred.

The option to review data extracts before publication was given to all participants, as well as the offer to withdraw from the research should they wish, up to 
the point at which data analysis began. The design of the semi-structured interview minimised the amount of personal information recorded. A consent document was given to each participant by way of initiating the informed consent process, which included specific information regarding ethical issues associated with the study; and as part of the recruitment process they received a document that included: the aims and focus of the study; details of the studies methods / methodology; participant inclusion criteria; and information relating to practical issues concerning the research process.

Hard copies of documents and audio recordings were held in locked storage, whilst electronic information was secured within an encrypted electronic storage device. The principal researcher was the only person able to access the secured information.

The research proposal, upon which this methodology was based, was submitted for formal scrutiny, and received full clearance from the University Research Ethics Committee (UREC) of Manchester University before the study was conducted.

Validity

A key criterion for evaluating the validity of qualitative research, identified by Stiles (1993), is the credibility of the researcher as a reflexive professional. The disclosure and expression of relevant data is dependent on the researcher's ability to develop a working alliance with participants (McLeod, 2003), and their ability to reflect upon, and monitor the research process. I am mindful that I have my own 'blind spots'; at times, in the interviews with participants, I have captured and challenged my own assumptions, especially when the participants would impart a theoretical concept from transactional analysis into our interview. Transparency was also communicated within the study by sharing my own clinical training and core theoretical modalities, philosophical values, and organisational pressures. Data collection and analysis procedures have been documented in detail, and participants were invited to comment on the accuracy of the transcripts derived from their interviews. Research supervision, and independent audits of my analyses by colleagues, have also been invaluable resources to heighten my reflexive processes.

\section{Results}

An overview of the superordinate themes that emerged from the data analysis, along with the themes that constitute them, are shown in Table 1. There were a number of themes that are not shown because they occurred in only one or two cases and the evidence for them was therefore considered too weak.

Complexity in Understanding Internet Addiction Confusion \& Complexity

Without exception, the theme of confusion and complexity in understanding Internet addiction emerged within every participant interview.

Jack: I think it's one of those ones that has been popularized within the media and I think that we've become... it's a bit grandiose... and I don't know who I think the "we" is, but I think it's become... you know, sometimes we have to ask ourselves some very difficult questions and... you know, How do I know the world is round and it's not flat?. It's one of those type questions. (2.24 -3.5).

Dora: And real is complicated, by its nature, and every... you know. There's general ideas about things. There's theories about things. But it may or may not... (laughs). I just don't think anything is black and white, and em... for me, it's exciting and I like it. (15.3 - 15.5)

Lucy: I think that it's not so much for me to... em... agree or disagree with a client's self-belief... I think it's about exploring by enquiry what their belief is, what they understand about it. Em... what the problem is for them, and what their desire is for change... (11.16 -11.18).

The results here suggest even within the transactional analysis modality, where therapists' share the same theoretical and philosophical foundations, there is a general struggle to conceptualise Internet addiction. However, some common ground began to emerge between two of the therapists, in their understanding of IA as an obsessive-compulsive or an anxiety based problem.

'IA' as an Obsessive-Compulsive Problem \& an Anxiety-Based Problem

It is possible to discuss the two themes referring to obsessive-compulsive and anxiety based problems together, as in many respects they are inextricably linked as obsessive-compulsive behaviours are largely driven by anxieties. Half of the sample showed confusion and complexity stemming from their experience that the nature and manifestation of an 'Internet addiction' can also share features with obsessive-compulsive disorder or other anxietydisorders, or at least could be understood through the lens of such disorders; thus bringing into question 


\begin{tabular}{|c|c|}
\hline Superordinate Themes & Themes \\
\hline Complexity In Understanding & $\begin{array}{l}\text { Confusion \& complexity } \\
\text { 'IA' as obsessive-compulsive problem. } \\
\text { 'IA' as an anxiety based problem. } \\
\text { Comorbidity or underlying pathological disorder. } \\
\text { Internet as medium for addiction. } \\
\text { Is Internet itself addictive? } \\
\text { Prevalence of IA behaviour }\end{array}$ \\
\hline Aetiology / Predisposing Factors & $\begin{array}{l}\text { Childhood origin } \\
\text { Script } \\
\text { Aloneness } \\
\text { The conflicted self. } \\
\text { A low self-esteem } \\
\text { Loss \& Instability }\end{array}$ \\
\hline Features \& Function & $\begin{array}{l}\text { Coping strategy } \\
\text { Searching \& collecting process (obsessions \& } \\
\text { compulsions) } \\
\text { Power, control and domination } \\
\text { Escapism } \\
\text { Intimacy and relationship difficulties } \\
\text { Loss of time } \\
\text { Discounting and splitting of self } \\
\text { Perception of stability }\end{array}$ \\
\hline Treatment & $\begin{array}{l}\text { Methods, techniques \& style } \\
\text { Vulnerability and safety of therapist } \\
\text { Support for therapist }\end{array}$ \\
\hline
\end{tabular}

Table 1: Summary of themes

whether IA is in fact a 'true' addiction, existing in its own right. Two of the participants referred explicitly to IA in these specific terms (Jack and Lucy), and more often than not, talked of 'anxiety' and 'obsessive compulsive' thoughts and behaviours simultaneously. O IA in these specific terms (Jack and Lucy), and more often than not, talked of 'anxiety' and 'obsessive compulsive' thoughts and behaviours simultaneously.
Jack: But my experience from looking at it and formulating it from a CBT perspective and thinking about it as a practitioner, in real practical ways, is that it seems to be more of an OCD. People have an impulsive... they have an obsessive thought which then results in impulsive behaviour... [ ] Em, and my sense is that it's a lot more OCD than it is addiction. And I believe that it's an impulsive thing, and I think that it's anxiety based... I think people ... it's often 
linked with perfectionism in my experience.... it's often linked with em... anxiety... [ ] That it's almost like there needs to be a pre-cursor of anxiety. (4.11 $-5.2)$

Lucy also views problematic Internet problems as a 'compulsive problem', and even frames the dynamic of relief of anxiety using the same words as Jack i.e. a process that enables the person to "self-sooth":

Lucy: ... my understanding is that there is an inability to regulate, either their own emotions, or regulate the affects of other. And that triggers a huge amount of anxiety which then needs to be regulated in some way, or distracted from, or disassociated from. $(6.2-6.4)$.

\section{Comorbidity or Underlying Disorder}

As reported by participants, given that features of such American Psychiatric Association defined disorders are shared by sufferers of IA, maybe this speaks more of a pattern of comorbidity or evidence of underlying disorders. The interviews with Jack, Kam and Lucy all revealed some evidence of comorbidity or an underlying disorder(s) in their work with clients struggling with Internet addiction.

Lucy: The first thought I had if I open that door in my mind was... deadness. There is something about their early experience where there has been neglect, but there has been a deadening atmosphere in the house, so like some kind of parental influence where they've been depressed, mental health issues of some kind, but a flat mental health issue, yes? (18.2 $-18.5)$

In working with the same client group, Jack also highlights the presence and importance of the underlying problems that give rise or accompany Internet addiction.

Jack: And you know, it's just a presenting issue. That's why I find it very kind of unthreatening. So it's just another one on the list isn't it.... like alcohol, this or that or.... I use porn or something like that.... and it's like.... Well, that doesn't particularly interest me, you know, it's the root cause... (19.11 - 19.15).

Obesity and food addiction is another comorbid factor evident in the interviews.

Dora: .... lot of them are overweight... and that's another whole addiction [ ]. Is it food addiction or is it used as an armour? [ ] come here ... go away. I want you, but you wouldn't want this would you. So go away, because I wouldn't know the first thing to do with you. I've got a fantasy about what I should do and I'm going to work towards that. But I'll make sure that I'm not... (30.23 - 31.18)

Kam: ... But when we talk about the social phobia... [ ] I think it's both... Em... I think it's both people with social phobia from the beginning. They can be autistic. They can have milder... or stuff like that. Erm, but also a consequence. $(9.22-10.2)$

Again, the complexity in understanding Internet addiction in its own right is brought to the fore, as Kam highlights that comorbid disorders often predate Internet addiction in a person's life, and may not necessarily be caused by it. In some instances, the opposite can be true, as suggested in all of the interviews.

\section{Internet as a Conduit or Medium for Addiction}

Closely linked to the emergent super-ordinate theme of comorbidity and underlying disorders is the notion that the Internet is a conduit or medium for addiction.

Kam: So I think... I think that it's not strange if we can find answers in those areas [the Internet]. That some people have greater or worse combination of drivers and injunctions [dysfunctional beliefs and behaviours], that connect to something, and I mean the Internet does everything. So it's really... every man's choice. So if you can get hooked in real life, of course you can get hooked on the Internet. (17.10 17.13)

Jack: ... And it does seem to be that that is quite vehicle specific. So there's something about the internet delivering that is quite important. $(6.2-6.4)$

Jack: ...No, so they've accessed that through the Internet and trawled through various people who will do that with them and then they will go and actually do that. $(29.16-29.18)$

\section{Addictiveness of the Internet}

A recurrent theme that emerged through two of the interviews refers to the question of whether the Internet is, in and of itself, addictive (as opposed to the content). This is closely related to, but has a slightly different emphasis to Internet as conduit for addiction, as opposed to addictive properties of the Internet. Arguably, this could be considered two sides to the same coin, which accounts why it has been highlighted here as a complicating factor. 


\section{Prevalence \& Pervasiveness}

Kam: (takes a deep breath)... I think it's a hard word because in a way we are all Internet addicted. All of our society is Internet addicted. We wouldn't work without the Internet. So it's a difficult word. (6.14 6.16)

Lucy: And it will also tell me [information about them], if that [Internet addiction] happens to be their addiction of choice in the given moment. Because we all have addictions and we all have ways to selfsooth or to try and regulate our feelings by ignoring them and focusing on the addiction... you know, the object of choice. $(12.6-12.9)$

Jack: I believe that people use the Internet excessively. But you don't say to somebody who drinks a lot, Oh you've got a pub addiction. (3.7 3.11)

Dora: Primarily, an inordinate amount of time spent [spent using the Internet]... and I think a lot of people, myself included... you know, what's addiction, and what's interest? You know, it's on a continuum. Yes, my husband and I can spend hours in the same room on our own machines doing stuff. But you can spend money [on the Internet] very easily, without realising it... $(8.11-8.15)$

Dora: ... people being able to trail where you've been. Is that different to when it used to be all in magazines? Or on videos? I don't know. Is that wrong? No, it's not wrong. (17.10 - 17.12)

Dora's perspective does raise the question, are we, as a society, pathologising what has been practiced for centuries simply because it is now accessed through a different medium, and leaves footprints? On the other-hand, is the ability to audit a person's thought processes and movements exposing what has been a problem since the proliferation of media?

Aetiology \& Predisposing Factors

Comorbidity came through as a theme for aetiology as well as for Complexity as above.

\section{Childhood Origin}

Script patterns formed in childhood / dysfunctional beliefs, or the development of dysfunctional schema in early childhood, is a significant predisposing or aetiological factor contributing to the development or manifestation of Internet addiction, being mentioned by each participant.
Kam: When you talk to the parents and talk to the family, talk to the youngster, and you ask, When do you think?, or, What happened? they say, Well probably it started earlier than I think... (19.15 -19.7)

The quote above, extracted from Kam's interview, neatly captured the shared experiences of all of the therapists. Many themes emerged across transcripts that related to a disturbed or disaffected childhood; upon closer analysis, a distinction started to emerge concerning the nature of the disturbance. It is perhaps most clearly explained using Lucy's borrowed conceptualisation of insecure attachment (Bowlby, 1969):

Lucy: To me, addictions are very linked with attachment issues [ ] if we are insecurely attached and our needs have not been met enough in maybe our childhood. (5.20)

Lucy: I have a hunch... if we think about childhood and there is one side where there is neglect, and [children] have been ignored and not [had] enough contact, and then there is another side of the trauma where [there] is contact but it is abusive contact, yes.... [ ] like being over-stimulated by something traumatic, or by being isolated and left alone [ ] my hunch would be that it's those that have been, if you like under-stimulated, ignored, neglected, left alone... [ ] that [they] would then have more of a leaning towards Internet addiction as opposed to other addictions. (6.22 - 7.12)

Lucy discerns between those children that were abused or traumatised, and those that were neglected. Using constructs employed by Kam, it is possible to discuss this distinction as "family type 1" (i.e. abused or traumatised), and "family type 2" (i.e. referring to those children who were neglected).

A common thread of childhood neglect resonates through each account. As Jack notes from his experiences, the neglect often starts at the earliest developmental "pre-linguistic" stages. Sometimes, the neglect would be subtle, in the families that "seem to have everything". When the parents are successful academically, or professionally, there is a risk that attentiveness toward meeting the child's needs diminishes. As can be inferred from Lucy's understanding below, clients are heavily defended against affect associated with neglect (e.g. terror and rage), and to continue repressing these emotions would seem the 'easiest' option, and to keep the addictive process alive: 
Lucy: [I]f the caretaker has not been in enough contact with the baby, the client then... my understanding is that there is an inability to regulate, either their own emotions, or regulate the affect of the other. And that triggers a huge amount of anxiety which then needs to be regulated in some way, or distracted from or dissociated from. $(5.20-6.4)$

\section{Loss \& Instability}

Loss and instability in childhood was a particularly prominent theme, and whilst it could be subsumed under the heading childhood origin, this was an especially recurrent theme with Kam and Lucy.

Kam: in the split it happens a lot to that kid. A lot of activities that finish, perhaps because they had to move or stuff like that. And then the computer becomes more important. (4.22 - 5.1)

Kam observes the central role the computer begins to play the young person's life, who is surrounded by losses resulting from parental separation. Arguably, this function can be as much about denial of the original loss as a method for resolving past hurts.

Kam: [the Internet] becomes some kind of support. It becomes also a place where you can be imaginatory. You can have the family that you want. You can play games where you can be the father or where you can be whatever you want. $(5.7-5.8)$

\section{Loneliness / Aloneness}

Loneliness and / or aloneness was a recurrent theme throughout the interviews with Jack and Dora. In the following extract from Jack's interview, there is evidence of an interplay between the aloneness his client experienced as a child, and the loneliness that has beset him in adulthood:

Jack: I'm thinking about one gay man I work with who is in his kind of forties, fifties... kind of very well adjusted in one sense... you know, a very senior social worker but he has got this very isolated story. This very kind of aloneness as a child... em... so he's been... he's very kind of quite schizoid.... (9.17 9.22)

Where individuals report an "isolated story", the interviews would indicate that the Internet provides a medium to at least partially meet their need for social and psychological contact. In his work with Internet 'sex addicts', Jack has noticed that many of his clients will spend hours surfing the net to find others to meet off-line, under the guise of intending to have sex:
Jack: And often the contact itself is very sexually minimal actually. Because l've worked with many men who just kind of talk to prostitutes or offer them a cup of tea... (30.8 - 30-10)

The risk of emotional vulnerability is moderated by Jack's lonely clients, by seeking company within 'professional' relationships. For some of Dora's lonely female clients, the arrangement is not so contractual. Dora's cites her clients, with a history of rejection and loneliness, who will search and network into the early hours of the morning in pursuit of actual offline social encounters.

\section{Low Self-Esteem}

Loneliness and low self-esteem issues are often inextricably linked. Dora notices that her lonely client fit a very familiar profile. As she reflects upon her practice, she notices that low self-esteem and loneliness are generally born out of looking physically different. This would especially be the case for her obese clients:

Dora: one of my mums, who spends five hours of the evening on a chat room... because they are very large and don't perceive themselves as particularly beautiful. (18.17 - 18.19)

Kam also observes, amongst his Internet addicted gaming clients, core dysfunctional beliefs underpin the addiction cycle, with many of his clients believing:

Kam: I am ugly...or I am outside. I am not one of the others. (12.10)

Besides serving as a haven to hide, the content of the Internet also provides us with a paragon of how we should be. Similarly, Jack believes, "pornography represents the unobtainable" (14.21).

Jack: I think it's more about [existential] life position... like I feel shit about myself... and so... in the porn I can have an ideal... I can fill in the gap that I'm looking for in order to feel okay. (14.7 - 14.10)

\section{A Conflicted Self}

Jack: ... the same theme seems to be emerging... it matters very much what the particular image is that's being sought [ ] the other partner is aware of that through discovery and then it's very kind of, often very distressed by that because they know that they [the partner] don't fit that fantasy image. (6.17-6.23)

The extract above represents a phenomenon that repeatedly manifested throughout Jack's interview. 
An individual can spend countless hours viewing Internet pornography without experiencing cognitive dissonance. In most people it is only when they have been discovered looking at particular images in the Internet that their problems begin.

Jack: Splitting is an absolutely key component of pornography for me. Which is something about my clients again, it's nothing to do with my real partner... it's a completely different feel. So, you know, I go and do this... I go and wank to porn... which is something over there, and then I exit that and I reenter real life. (14.18 - 14.24)

Functions \& Features of Internet Addiction 'Function' refers to how the addiction serves to ostensibly meet the needs of the user of the Internet, albeit in a dysfunctional or pathological manner, thus reinforcing unhealthy patterns. In this sense function could also be understood as dysfunction. 'Feature' refers to how the addiction manifests, is experienced by the user, or is identified by others.

\section{Coping / Searching \& Collecting}

Jack: There is that sense of belief that I have to engage in this behaviour in order to manage or survive $(5.15-5.17)$

The quote above, extracted from Jack's interview, neatly captures what it is like for many of the afflicted clients referred to throughout this research. As Jack reflects on his practice, he empathizes with his "typical" client's struggle with addictive "behaviour". At best, their behaviour in using the Internet is used a coping mechanism to manage in life; at worst, is it used to defend against a perceived existential threat, to their very survival in the world.

In reconsidering her experience of family members (as well as clients) who use the Internet to cope, Lucy notes:

Lucy: I think this... this collecting and searching... collecting and searching... collecting and searching... as if this site... or this piece of information is not soothing... but it's the search... it's the search that does the soothing... it's the search that distracts from the discomfort the most... So the search is actually the addiction... not the finding of [content] $(16.20-16.23)$

Lucy: So I hadn't thought that before. It's like searching for the home. [ ] Yes. And a final resting place, yes? (17.7-17.17)
Other therapists report similar findings. In discussing clients that use the Internet to find actual sexual partners, Jack notes:

Jack: [ ] it's not just a question of going on a supermarket website and choosing someone, you know. There's a kind of excitement to the process of the choosing. So there's something in that process which matters in itself. $(30.2-30.6)$

However, with other clients, the 'searching as soothing' function is not so clear cut:

Jack: So there is a kind of drilling process and accessing porn where men seem to be looking for the specific right image, or the right video or whatever it is, and so trawling through material and then diving deeper into stuff that kind of hooks them more... $(5.21-5.23)$

For other clients the Internet serves as a coping mechanism to reconnect with 'deadened' parts of their personality. In recounting her experience of working with clients who have survived childhood neglect, resulting from living in a home environment with a depressed parent(s), Lucy notices how the child learns to cope:

Lucy: [T]hey learn this passivity and stillness... [ ] the only stimulation that they can... that they cope with, is this boxed in stimulation there. Not with people, [no] contact around, yes? (18.12 - 18.14)

After 'boxing in' and deadening stimulation, the Internet become the safest way to access, tolerate and manage their feelings. Conversely however, for many of Kam's Internet gaming addicted clients the computer become a place to cope with the seemingly intolerable feelings that can emerge off-line. Rather that the Internet being a medium to access feelings, it functions as a vehicle to avoid or cope with unpleasant feelings:

Kam: ... you notice the shitty world you live in... [ ] And then you tend to want to go to the computer to forget. $(19.4-19.7)$

\section{Power, Control \& Domination}

Power, control and domination were recurrent themes within and across cases. Participants noted the significance these themes have in terms of defining a user's Internet addiction, and the function the Internet serves in meeting their perceived need for power and control. This was a particular 
characteristic of Internet gaming addiction, where the Internet served to apparently reconcile the power imbalances that develop between family members.

Kam: You can be strong and you can fight back... [ ] And also... a place where you can be good. [ ] Because often, it has become a problem in school... that you don't achieve in school. But in the games you can achieve. $(5.12-5.19)$

The results suggest the need for a sense of power and control was also found in users attracted to other sub-types of Internet addiction. Similar dynamics were found underpinning cyber-sex addictions:

Jack: And I suppose it's something about I cannot tolerate difficult feelings or complexity in a relationship, so what I do is I access porn where I can have something but not the others... what can I have? Physiological relief, I suppose, a sense of potency. [ ] A lot of porn is about power and control, without having to pick up the complexity of relationships. $(13.18-14.2)$

\section{Intimacy \& Relationship Difficulties}

The function and features of power and control, within IA, overlap with another pervasive theme of intimacy and relationship difficulties. I would like to highlight an interesting power and control dynamic within Dora's clinical experience. In working with disaffected young mothers, primarily in an off-line group format, she works simultaneously with the same clients in an on-line Internet 'chat room'. Dora has noted how her clients will communicate more freely with her group members on-line: It would appear, in these instances, for these clients, the Internet can serve as a mechanism to control the amount of intimacy, much like a volume dial on a radio.

Dora: You're there in real life, and I just think, So you're hiding on purpose, knowingly. And yearning for intimacy... Why (laughs). I couldn't guess as to why. [ ] My guess is it's less scary somehow. (12.1012.15)

Jack reflects on a similar pattern:

Jack: There might be some kind of relational difficulty or something like that and then the belief will be something like, "Nobody meets my needs, and I can't tolerate that or ask for what I want", and there will be a discount at that moment... [ ] And so then, what he'll do is, he'll access pornography to meet his needs. (26.12-26.19)

\section{Escapism}

It can be argued that escapism is another form of coping. Certainly, within these findings, there is a degree of overlap between all of the higher-order concepts. For instance, Jack notes, "A lot of porn is about power and control, without having to pick up the complexity of relationships" (14.1). Clearly, this data speaks to at least two higher-order concepts. However, given its repeated emergence as a term or concept, frequently and explicitly used by $75 \%$ of the sample, it warrants a particular focus.

Jack: So people use laptops for work... [ ] with the flick of a switch, they can then access porn at the same time. So I think there's something about that switch between work salient distress, you know, something linked to their work that is salient, and then moving into this [virtual] world...(22.6- 22.11).

Jack: Choosing not to think, which is a massive one. [ ] So I think, in the process it's that huge thing about choosing not to think and running away, you know. (37.4-37.12).

Kam: It becomes also a place where you can be imaginatory. You can have the family that you want. You can play games where you can be the father or where you can be whatever you want. $(5.6-5.9)$

Kam defines how he understands Internet use as different from other forms of escapism:

I mean... it's a lack of something when you drink too much, or when you are addicted to drugs also... but the difference here... is your experience... This drug... this Internet drug... it's a slow drug... You can sit there and time stops to exist. $(7.3-7.5)$

It's only when you stop playing. [ ] that you notice the shitty world you live in... [ ] And then you tend to want to go to the computer to forget. $(19.2-19.7)$

Lucy: And l've seen that with clients and I also see that in myself, too [ ] it's like the (Internet) search that it causing me to forget about my own stuff, my own feelings, my own process, yes? (17.10 - 17.12)

\section{Treatment}

\section{Methods, Techniques \& Style}

The theme concerning method, technique and style of the therapist emerged across every case. This is not surprising given that my semi-structured interview was partly informed by predetermined 
questions concerning the therapist experience and understanding of how therapist participants work with people struggling with internet related problems. A common, clinical perspective began to emerge across participants, whereby the 'Internet addiction' was treated as symptomology.

Jack: And you know, it's just a presenting issue. That's why I find it very kind of unthreatening. So it's just another one on the list isn't it... like alcohol, this or that or.... I use porn or something like that... and it's like... Well, that doesn't particularly interest me, you know, it's the root cause... (19.11 - 19.14)

Kam: I mean, as I said before... now it's Internet... it could have been something else. 14.20

Lucy: I guess I come at it from a different lens... a different perspective. To me, whatever the addiction is... whether it's Internet addiction or drugs or alcohol or whatever... [ ] For me, that's the solution. So I don't actually explore and... I don't make that my focus. [ ] my focus is... [ ] The Child (ego state) and the attachment needs inside the client. $(9.17-10.5)$

Each of the quotes capture the therapists' understanding of Internet addiction as symptomology of an underlying "root cause", the implication being that the Internet is the clients' addiction of choice. Whilst acknowledging the addiction as a "presenting problem", the therapists' treatment direction involves a shifting focus towards, for example, the developmental attachment deficits of the client.

Lucy: I think, that if someone chooses the Internet, then it tells me that they're... em... (pauses)... I can't think of the word... something about their interrelational process... that contact in relationship is very, very difficult. So that... that... as soon as somebody says that, then I know that... em... I will attune to their capacity to be in relationship, which may be a momentary contact, eye contact or looking. You know, that kind of stuff. [ ] So, I will evaluate as I go, I will sense, you know, how... how much contact they can sustain. $(12.11-12.19)$

Lucy will attune to the developmental "attachment needs inside the client, informed by an ongoing process of feedback and evaluation based on relational and behavioural observations within the relationship. With a keen focus on relational working, Lucy will extensively employ the services of metaphor in aiding her understanding of working with the client:
Lucy: It's engaging with somebody's Child (ego state), but through the third person as it were, through the metaphor (14.21)

Lucy: The visuals that I get is... thinking about [ ] It's like the equine therapy... [ ] he waits for the horse to come to him, by being on side. So you are not confronting... $(14.3-14.6)$

It is not clear whether this is done explicitly, by sharing the metaphor with the client, or whether it is simply using a technique that enables her to personally connect with the vulnerability of the client.

Lucy will also use metaphor as a means by which she enters and understands the transference in her therapeutic relationships. By using her own countertransferential response as a therapeutic tool to "attune" to her clients' developmental attachment deficits, she can then moderate the levels of intimacy she provides.

Jack's approach would largely incorporate cognitive behavioural interventions, including working with the client in "heightening" their awareness of triggers, and exploring their dysfunctional beliefs:

Jack: [T]here's a trigger of which we make a meaning which is then not accurate. So it's meaningmaking. (35.21)

Jack: ...so then really heightening that interpretation of that thinking. (36.6)

By working with clients in identifying the unhelpful beliefs they hold, and the affect this has on their emotional and physiological states, Jack will guide them through a process of assessing the "validity" of their beliefs, followed by a series of interventions that aim to support the client in identifying healthier ways of meeting their needs.

Like Lucy, Dora's treatment focus is on the relational components of the relationship. "Well, I have a great nuance to people's Child (ego state). So I would want to find out where that got missed somewhere." (20.15) By working with the client in locating the source of the developmental attachment deficits, the focus can then shift towards supporting the client in the therapeutic healing process. In this quest, both Dora and Kam would employ a group therapy modality. By way of reaching those clients that spend an "inordinate" amount of time on-line, Dora had set-up a forum where group members could interact. However, Dora seems confused and / or conflicted about her role within the on-line facet: 
Dora: I didn't want them to think that I was... em spying... I wanted them to know that I... and I put... you know, There's been dreadful rain. Remember everyone to bring your wellies or whatever... I put functional stuff on there. Here and now stuff on there. So they know I'm there. $(13.10-13.14)$

Kam sees his clients in groups, as individuals (the addicted user), and with their parents, and also works with parents alone.

Kam: $(T)$ he family is a family, and the family has a process, I mean it's also a way of saying and making it clear that the client is not the problem. The system is the problem and we work with the system. (13.5 13.7)

In working predominantly with the family as a "system", he works creatively, flexibly and "intuitively", and does not adhere to a specific treatment plan or protocol. For Kam, Internet addiction in a result of dysfunction with the family system, although he recognises that patterns in the way he works have emerged which would suggest a familiar treatment direction. Like Jack, he finds himself working with dysfunctional beliefs in the early stages of therapy, at the cognitive level:

Kam: And it's also a lot of decontaminations. Because the bad memory often becomes a contamination... [ ] I am ugly...or I am outside, I am not one of the others...or stuff like that... (11.21 12.7)

At the same time, treatment interventions will involve reappraising the quality of the life the young and addicted Internet gamer is experiencing, and Kam will invite these clients to hone their focus on a richer and more fulfilling experience.

\section{Vulnerability \& Safety of the Therapist}

Throughout three of the participant interviews, the theme concerning the vulnerability and safety of the therapist became apparent. Within each case analysis they had emerged under different headings, i.e. how I had labelled them at the time of analysis: impact on therapist and safety, therapist vulnerability and challenges, and therapist as a person. The results are presented here under the composite heading, vulnerability and safety of the therapist.

It has been noted earlier how Jack often understands Internet addiction as an obsessive compulsive disorder (OCD); he highlights the caution necessarily involved in his working with those afflicted.
Jack: [Y]ou know, people kill themselves on the back of it quite often... that we need a very, very specific kind of treatment methodology and conceptualization... [ ] And I kind of see porn, Internet porn usage, as kind of in and around that in some way. $(18.12-18.20)$

He also emphasises the dangers of providing interventions that are not evidence-based. Given the intense personal investment involved in working psycho-dynamically, perhaps it also feels safer for Jack to work at a cognitive-behavioural level, in an attempt to avoid personally processing the meaning of transferences, at the experiential level:

Jack: I think often people are really struggling with a lot of shame and I get very bored with that... [ ] It's like I kind of... the way... I feel a bit like a Goulding [a therapist that works 'outside' of the transference] in some way, you know. Like your pathology bores me... it doesn't... I'm not too worried about it... you know... and l've seen it and heard it all. And I kind of feel like... I'm sure I haven't... but I kind of feel like I almost have... $(28.12-28.19)$

The above quote would suggest that Jack is experiencing some inner conflict around the familiar feelings that emerge in his therapeutic relationships.

Lucy's approach to working with clients often involves 'stepping into' the transference:

Lucy: it [working with Internet addicted clients] triggers stuff for me, because I had the experience in childhood, not of... not so much of trauma and chaos, although there was chaos... but of noncontact. My mother is schizophrenic so I didn't have enough contact. $(8.6-8.8)$

Lucy: Sometimes I'm left... transferentially with some of the deadness and the flattening. [ ] Em... Sometimes I experience being rendered passive in the room. (22.10 -22.13)

She recognises her own pro-active countertransference (i.e. what she projects into the world from her past, her presuppositions), resulting from a lack of psychological and physical contact from a schizophrenic mother. The following quote illustrates how Lucy skilfully "guards" against working in the transference, because of her own "live material", and instead will mindfully work with the transference:

Lucy: With those kind of clients... em... in my experience... the way that I work with them at this current period in time, is that I will work with the transference but I will guard against going into it. [ ] 
Because I think that I have my own live material, em... I was quite abusive to my son when he was growing up. I was a single parent and I think there's a part of him that still wants to "Fuck off his Mum"... As well as me... em... [I'm] probably still needing to do that with... my Dad, actually. I don't know actually... I think maybe... yes... actually I hadn't thought of that before... If I get to grips with the stuff with my dad probably my son will feel more free than me, but anyway. Yes. So I will have the ability to shut down and be strong. I will deliberately ' $\mathrm{Be}$ Strong' around that kind of client. So that they are... like they are... it's like they are hitting off of me. (24.2 -24.13)

\section{Support for the Therapist}

Receiving appropriate supervision for Internet addiction work can be a challenge within itself:

Dora: And I have to say that, as a therapist, l've struggled to... I've struggled... I feel like I've struggled to get the level of support by way of supervision [ ] commensurate with the stuff that I'm dealing with. $(25.7-25.12)$

Dora: [The] supervision that I have is more concerned with the outcome (25.16)

Dora is clear as to what she needs in supervision, somebody to help her "recognise and monitor" client material, as well as the provision of empathy and emotional support for her, as a therapist, as a person. Without the appropriate level of supervisory support the vulnerability of the therapist and that of the client will remain in jeopardy. Dora notes that to support the therapist as an effective provider of treatment to people struggling with Internet addiction, supervision is just part of the "scaffolding" of support:

Dora: ....as long as you have the scaffolding, ethics, supervision, therapy... the things that are there for this reason. If you've got the scaffolding, within that, you can be creative. $(15.7$ - 15.9)

Kam adds 'research' to the "scaffolding" of support:

Kam: But I don't have years of science to rest the work on. And that makes it frustrating. (15.21 15.22)

Kam: ...many of those services are funded by the gaming industry. So there are not much research that are... free from funding (16.10)
To Kam, the source of his 'frustration' at not feeling supported by research is that the studies that do exist, concerning Internet gaming, are heavily funded by the gaming industry, exposing a conflict of interests. What Kam seems to be inferring is that research questions, and research designs, are biased and that there would be little motivation to publish controversial findings. When research is conducted purely in the interests of clinical curiosity, and without political bias, organisations can turn a 'blind eye'. At least this was Jack's experience, after conducting a substantial piece of clinical research into Internet pornography addiction:

Jack: And they couldn't hold it in any way... because that's not an NHS problem...we don't have lads coming to their GP saying, "I can't stop wanking", kind of thing. So then there could not be a conceptualization, which I thought was really interesting. [ ] So, it's something political in it about we see what we want to see and we don't see what we... So I suppose what I would say is my private practice is full of this... full of it... like a real percentage of my number of clients I see is this...You know, like... I don't know $17 \%$ or $20 \%$ or something. But then in the NHS I see clients all the time, three days a week, and it never appears. [ ] Em... it's clearly not... I dunno.... not debilitating enough or something, politically. [ ] It's not pathological enough or something... but it really, really distresses people... [ ] It really distresses people doesn't it? I don't know what your experience is, but you know, really, really distressed. $(42.3-43.2)$

In this instance, the NHS Trust he was employed by would not "hold" his paper, and its findings. Jack identifies this as a "political problem" given his own experience of working with individuals presenting to his private practice with Internet pornography addiction (" $17 \%-20 \%$ "), compared to his experience of working within NHS services, where "it never appears".

\section{Limitations}

A number of methodological considerations emerged as a result of this study.

The methodological approach chosen for this study enabled a rigorous exploration of the experiences and understanding of therapists working with clients struggling with Internet addiction; the idiographic approach of IPA is ideally suited for "scoping out" novel areas of research. However, because of the idiographic approach it is not possible to simply generalise the findings from this particular 
study to all therapists working with Internet addiction. Instead, the aim of the study was to focus on the idiosyncratic nature of the experience and understanding of psychotherapists working from a specific modality, which might have otherwise been overseen within positivist approaches. Thus, the unique and contextualised experience of particular therapists in particular circumstances is of paramount ontological concern (Smith, 1996; Jarman, Smith \& Walsh, 1997; Smith, Flowers \& Larkin, 2009).

A small sample was purposefully chosen (Smith, Flowers \& Larkin, 2009), and recruited through the European Association of Transactional Analysis (EATA). It is therefore probable that this research only captures the understanding and experiences of therapists with a pre-existing interest and expertise in the area I have chosen to study.

Measures were taken to select a fairly homogeneous sample (Smith, Flowers \& Larkin, 2009 , p. 48) in terms of professional competence and transactional analysis as the main approach. The requirement that participants also regard themselves as integrative may account for the radical differences that emerged between them.

In terms of cultural context, three of the participants were born, raised, and developed their therapeutic careers chiefly in the UK, whilst the fourth participant has lived and worked in Germany all of his life. Findings, therefore, may also reflect differences from having been brought up in different cultural contexts. It is also important to note that whist proficient in speaking English, the German participant was not interviewed in his mother-tongue (i.e. German), which may have influenced his and my understanding and interpretation of questions and responses.

Personal experiences of addiction were not addressed directly. Whereas participants were very forthcoming about their clinical experiences; only two gave personal accounts of their own experience of addiction, and how this impacts on their practice. It might have been useful to have explicitly enquired into therapists' personal relationship with addiction. As it transpired all therapists within the sample believed that "we are all addicts" to some degree.

It is important to consider researcher theoretical and philosophical stance, especially so given the interpretative, double-hermeneutic nature of the IPA methodology (Smith, Flowers, \& Larkin, 2009). It is inevitable that this will have influenced data interpretation, despite my best efforts to bracket
(Husserl 1970) this experience and understanding. I found it helpful to remind myself that $I$ am an experienced therapist, and have undertaken many years of personal therapy. This has helped me, as much as it can, heighten my skills at 'reading' my own counter-transference, and 'containing' it (even though, of course, this is never entirely possible). With the help of my personal journal, close supervision and audits by colleagues, I have felt relatively confident that I have taken sufficient steps towards appropriately bracketing (and embracing) my own particular views, and therefore centregrounding the experience of my participants.

Whilst conducting the interviews, I became aware of dynamics and processes unfolding between myself, as interviewer, and the participants. It became clear that this information was vital, as it served to provide even richer insights into the participants' phenomenological experience and understanding of Internet addiction. However, it may also have impacted on the results.

The first of these was the level of personal disclosure. Participants shared that in many cases they were aware that their own experiences impacted on their work, such as their own seeking sexualised chat through the internet, being raised by a schizophrenic mother, or surviving in children's homes as a teenage prostitute.

Another factor was the parallel process (Cornell \& Landaiche, 2006) of which I became aware of during the interviews. As an experienced psychodynamically trained psychotherapist I could not help but notice (along with some of my participants) the powerful dynamics at play as experience and understanding of Internet addiction were discussed. These represented another rich layer of data concerning what it is like to work with Internet addicted clients. For example, an intriguing insight into the unfolding parallel process occurring between myself and Jack arose following a discussion exploring the predominantly cognitivebehavioural way in which he believes he works with his clients. The more he reflected on his therapeutic work with men addicted to Internet pornography use, the more he realised he does in fact also work in a very relational and existential manner.

Whilst accounting early developmental factors, Jack's sentences became fragmented, and punctuated with pauses. He realises that he has flitted across various theories, explanations, and treatment interventions, and feels "all over the place". At this stage I feel a very strong urge to 'rescue' him from feeling "all over the place", and in 
attending to my growing anxiety I decide to share that this is my first research interview for this study. Perhaps feeling more contained within the interview process, Jack now develops a better understanding of his experience within the interview. Jack's insight and sense-making of his experience in the transference, and my impulse to 'rescue' the "unheld baby" would appear to neatly capture some of the "really complex" dynamics that occur between himself and his Internet pornography addicted clients. At the level of treatment, Jack also concludes that a purely cognitive-behavioural problem-solving approach is not sufficient in accounting or attending to early attachment deficits that are often the source of Internet addiction.

As another example, within my interview with Lucy I noticed many examples of parallel processes within the interview. Lucy uses her own countertransference to attune to her clients' struggle to regulate affect, and the personal challenges that poses for her. My experience of being in the interview felt very much like "we're finding our own water-level together". Like Lucy's experience of being with her clients, at times I feel "pushed away" by her, wondering maybe I have got "too close" too soon. I believe that, at this early stage in the interview, I have understood, and countertransferentially received (at the level of affect), what it feels like for her in working with her Internet addicted clients. Later, I experience a particularly powerful sense of understanding the dynamics unfolding between Lucy and I, and therefore the ambivalence to connect she reports in her work with her clients. It is not just a part of Lucy that is "desperate for contact and connection" with her clients; Lucy is also met by a similar ambivalence within her clients.

In a recent IPA study by Westland and Shinebourne (2009) exploring self-deception and the therapist, the experiences and understandings of therapists working with clients they describe as "self-deceptive" was explored. The writers conclude their findings with the following insights: therapist experience feelings of discomfort, frustration and anger, and a sense of incompetence when working with such clients; and that there was "some awareness of the possibility of the therapist's own self-deception" ( $p$. 398). The Westland and Shinebourne (2009) findings are of particular interest, given the findings I have just discussed regarding parallel process, as it would seem plausible that parallel processes could have emerged within the interviews with the researchers. Such parallel processes can provide a rich layer of data when exploring phenomenological concerns, although they may also be a limitation in terms of the impact on the researcher's ability to analyse data.

\section{Conclusion}

The study has sought to answer the question, "What is the transactional analyst psychotherapists' experience and understanding of working with clients who present with Internet related problems?" Four higher-order concepts emerged within this study. They concerned participant understanding and experience (and interviewer interpretation) of the complexity of Internet addiction (IA), aetiological and predisposing factors, functions and features of IA, and treatment factors. The findings spoke of the therapists' perceptions of the functions, features and factors of Internet addiction, based upon their experience of working with clients struggling with this phenomenon. Key conclusions and recommendations have been as follows.

The Internet was understood by participants as a conduit or medium for addiction. They perceived there to be evident a high prevalence of an underlying or pre-existing 'disorder', and especially features associated with depression or obsessive compulsive disorder.

All of the participants believed in the existence of childhood aetiological roots which underpinned comorbidity with Internet addiction. They believed that attachment difficulties in childhood would often predispose individuals to issues around loneliness, low self-esteem, control, loss and instability, and cognitive dissonance later in life.

Participants held the belief that clients presenting with Internet addiction used the Internet as a coping mechanism, or a device to escape the world. Typically, therapists' clients would struggle with intimacy and experience relationship difficulties.

Participants understood that clients with a history of depression or low mood would often use the Internet to access repressed feelings. They often experienced a relationship manifested between depression, low self-esteem and escapism which were specific and common factors that often contributed to the development of Internet addictions within their clients.

A systematic review of existing research, and future quantitative studies, could specifically evaluate the strength of the relationship, noted by the participants, concerning depression, low-self-esteem, and escapism within Internet addicted clients. Further empirical research is particularly recommended to explore how these factors may predispose 
individuals to developing sub-types of Internet addiction, and especially in the context of historic childhood abuse and / or neglect.

Participants held the view that Internet addiction is inherently complex in nature, and its aetiology is not so clear, and therefore requires a high level of skill from the therapist wishing to work with such client symptoms. Knowledge, skill and expertise could be developed further in specific trainings concerning childhood attachment difficulties. Integrating a psychodynamic approach, or being aware of transference processes, is likely to enhance treatment effectiveness, and would help safeguard both clients and therapists from counter-therapeutic interventions.

Conducting an interpretive phenomenological analysis study demands a very high degree of personal and professional investment. I noted above the importance, significance and challenge of 'bracketing'. As experienced as I consider myself to be as a therapist, the challenge to contain my preconceptions, presumptions and countertransferential reactions will remain. One of the key concepts that emerged within the study concerned the complexity and confusion surrounding Internet addiction. Immersing myself in the confused and / or confusing 'worlds' of the participants in exploring their confused experiences and understanding required a great deal of resilience and personal selfawareness. The picture is further complicated by my finding that childhood disaffection and insecure attachment often underpin Internet addiction, which could feed into the parallel process. Research into IPA researcher anxiety tolerance would be a fascinating endeavour, especially with those that are whole-heartedly and thoroughly invested in the process.

Dr Matthew Shorrock is a Provisional Teaching \& Supervising Transactional Analyst (Psychotherapy) and Chartered Counselling Psychologist, and can be contacted on info@themindinstitute.at

He submitted the material on which this work is based as a thesis to the University of Manchester School of Education as part of the Professional Doctorate in Counselling Psychology/

\section{References}

Aboujaoude, E., Koran, L. M., Gamel, N., Large, M. D., \& Serpe, R. T. (2006). Potential markers for problematic Internet use: A telephone survey of 2,513 adults. CNS Spectrum, The Journal for Neuropsychiatric Medicine, 11(10), 750-755
Acier, D., Kern, L. (2010). Problematic Internet use: Perceptions of addiction counsellors. Computers \& Education 56, 983-989.

Altman, D. G., Schulz, K. F., Moher, D., Egger, M., Davidoff, F., Elbourne, D., et al. (2001). The revised CONSORT statement for reporting randomized trials: Explanation and elaboration. Annals of Internal Medicine, 134, 663-694.

Bowlby, J. (1969). Attachment and loss: Volume 1. Attachment, New York: Basic Books.

BPS. (2010) Code of Human Research Ethics. Retrieved on $11^{\text {th }}$ October, 2012, from

http://www.bps.org.uk/sites/default/files/documents/code_ of_human_research_ethics.pdf

Byun, S. et al. (2009). Internet Addiction: Metasynthesis of 1996-2006 Quantitative Research. CyberPyschology \& Behavior (12), 203 - 207.

Caplan, S. E. (2002). Problematic Internet use and psychosocial well-being: Development of a theory based cognitive-behavioural measurement instrument. Computers in Human Behaviour (23), 265-271.

Chirban, J. T. (2006). Integrative Strategies for Treating Internet Sexuality : A Case Study of Paraphilias. Journal of Clinical Case Studies, (5), 126-141.

Churchill, S. (2000). Seeing through 'self deception' in narrative reports: Finding psychological truth in problematic data. Journal of Phenomenological Psychology, 31, 44-62.

Cornell, W. \& Landaiche, M. (2006). Impasse and Intimacy: Applying Berne's Concept of Script Protocol'. Transactional Analysis Journal. (36)3.

Cui, L. J., Zhao, X., Wu, Z. M., \& Xu, A. H. (2006). A research on the effects of Internet addiction on adolescents' social development. Psychological Science, 1, 34-36.

Davis, R. A. (2001). A cognitive behavioural model of pathological Internet use. Computers in Human Behaviour (17), 187-195.

Davis, R., Flett, G., \& Besser, A. (2002). Validation of a new scale for measuring problematic Internet use: Implications for pre-employment screening. CyberPsychology and Behavior, 5(4), 331-345.

Etherington, K. (2004). Becoming a Reflexive Researcher. London: Jessica Kingsley.

Ferster, C. B., \& Skinner, B. F. (1957). Schedules of reinforcement. New York: Appleton-Century-Crofts.

Goldberg, I. (1996). Internet addiction disorder Retrieved 9th March, 2011. 
Goulding, R. \& Goulding, M. (1976) Injunction, decisions and redecisions. Transactional Analysis Journal, 6(1): 418.

Goyette, M., \& Nadeau, L. (2008). Utilisation pathologique d'Internet: Une intégration des connaissances. Alcoologie et Addictologie, 30(3), 275-283.

Greenfield, D. N. (1999a). Virtual addiction: Help for Netheads, Cyberfreaks, and those who love them. Oakland: New Harbinger Publications.

Greenfield, D. N. (1999b). Psychological characteristics of compulsive Internet use: A preliminary analysis. CyberPsychology \& Behavior, 8(5), 403-412.

Greenfield, D. N. (2011). The addictive properties of Internet usage. In K. S. Young \& C. N. Abreu (Eds.), Internet Addiction - A Handbook and Guide to Evaluation and Treatment. New Jersery: John Wiley \& Sons.

Heidegger, M. (1962 /1967). Being and Time. Oxford. Blackwell.

Hshieh, S., \& Srebalus, D. (1997). Alcohol treatment issues: Professional differences. Alcoholism Treatment Quarterly, 15, 63-73.

Humphreys, K., Greenbaum, M., Noke, J., \& Finney, J. (1996). Reliability, validity, and normative data for a short version of the Understanding of Alcoholism Scale.

Psychology of Addictive Behaviors, 10, 38-44.

Husserl, E. (1970). The Crisis of European Sciences and Transcendental Phenomenology (D. Carr, Trans.). Evanston. Northwestern University Press.

ITA. (2008). Code of Ethics and the Requirements and Recommendations for Professional Practice. Retrieved 11th March, 2011

Jarman, M., Smith, J. A., \& Walsh, S. (1997). The psychological battle for control: a qualitative study of health care professionals understanding of the treatment of anorexia nervosa. Journal of Community \& Applied Social Psychology, 7, 137 - 152.

Kelly, T. and Strupp, H. (1992) Patient and therapist values in psychotherapy: Perceived changes, assimilation, similarity and outcome. Journal of Consulting and Clinical Psychology, 60, 34-40.

King, D. L., Delfabbro, P. H., Griffiths, M. D., Gradisar, M. (2011). Assessing clinical trials of Internet addiction treatment: A systematic review and CONSORT evaluation. Clinical Psychology Review, 31, 1110-1116.

Kuss, D.J. \& Griffiths, M.D. (2011). Excessive online social networking: Can adolescents become addicted to Facebook? Education and Health, 29. 63-66

Liu, C. Y., \& Kuo, F. Y. (2007). The study of Internet addiction through the lens of interpersonal theory. Cyberpsychology and Behavior, 10(6), 779-804.
McLeod, J. (2003). Doing Counselling Research (2nd ed.). London: Sage Publications.

Morahan-Martin, J., \& Schumacher, P. (2000). Incidence and correlates of pathological Internet use among college students. Computer and Human Behavior, 16, 13-29.

Morahan-Martin, J. (2005). Internet abuse: Addiction? disorder? symptom? alternative explanations? Social Science Computer Review, 23(1), 39-48.

Moyers, T., \& Miller, W. R. (1993). Therapists' conceptualizations of alcoholism: Measurement and implications for treatment decisions. Psychology of Addictive Behaviors, 7, 238-245.

Najavits, L. , Crits-Christoph, P. and Dierberger, A. (2000) Clinicians impact on the quality of substance use disorder treatment. Substance Use and Misuse 35 , 2161-2190.

Najavits, L., Griffin, M., \& Luborsky, L. (1995). Therapists' emotional reactions to substance abusers: A new questionnaire and initial findings. Psychotherapy: Theory, Research, Practice, Training, 32, 669-677.

Niemz, K., Griffiths M., Banyard P. (2005). Prevalence of pathological Internet use among university students and correlations with self-esteem, the General Health Questionnaire (GHQ), and disinhibition. Cyberpsychology Behaviour 8(6), 562-570.

Oxford English Dictionary Online http://www.oed.com/ accessed 9 March 2013 REFERENCES

Ricoeur, P. (1970). Freud and Philosophy: An Essay on Interpretation. New Haven: Yale University Press.

Ricoeur, P. (1981). Hermeneutics and the Human Sciences. Ed. John Thompson. Cambridge: Cambridge University Press.

Rideout, V. J., Foehr, U. G., \& Roberts, D. F. (2010). Generation M2: Media in the lives of 8 to 18 year olds. Retrieved 16th March, 2011.

Shapira, N. A., Goldsmith, T. G., Keck, P. E., Jr., Khosla, U. M., \& McEIroy, S. L. (2000). Psychiatric features of individuals with problematic internet use. Journal of Affective Disorders, 57, 267-272.

Shinebourne, P., \& Adams, A. (2007). Therapists' understandings and experiences of working with clients with problems of addiction: A pilot study using Q methodology. Counselling and Psychotherapy Research, 7(4), 211-219.

Shorrock, M. (2011). The philosophical challenges within counselling psychology: Can Egan's Skilled Helper Model help? Counselling Psychology Review (26), 3, 63-74.

Shorrock, M. P. (2012a). The pragmatic case study of Ed - A man who struggled with Internet addiction.

Counselling Psychology Review (27), 2, 23-35. 
Shorrock, M. P. (2012c). Is Defusion Decontamination? An examination of seemingly similar concepts and methods used in Acceptance Commitment Therapy and Transactional Analysis. Unpublished.

Shorrock, M. P. (2013). Why people become Internet addicted: A qualitative meta-synthesis of studies that explore aetiology, predisposing factors and other antecedents to Internet addiction. In press.

Smith, J. A. (1996). Beyond the divide between cognition and discourse: Using Interpretive Phenomenological Analysis in Health Psychology. Psychology and Health (11), 262-271.

Smith, J. A. (2007). Hermeneutics, human sciences and health: Linking theory and practice. Journal of Qualitative Studies on Health and Well-Being.(2), 3-11.

Smith, J. A. (2011) Evaluating the contribution of interpretative phenomenological analysis Health Psychologyv Review 5: 19-27

Smith, J. A., Flowers, P., \& Larkin, M. (2009). Interpretive Phenomenological Analysis: Theory, Method and Research. London: Sage Publications.

Smith, J. A., \& Osborn, M. (2003). Interpretative phenomenological analysis. In J. A. Smith (Ed.), Qualitative psychology: A practical guide to methods (5180). London: Sage.

Soule, L., Shell, W., \& Kleen, B. (2003). Exploring Internet addiction: Demographic characteristics and stereotypes of heavy internet users. The Journal of Computer Information Systems, 44(1), 64-73.

Stiles, W. B. (1993). Quality control in qualitative research. Clinical Psychology Review(13), 593-618.

Thatcher, A., \& Goolam, S. (2005). Defining the South African Internet "Addict": Prevalence and biographical profiling of problematic Internet users in South Africa. South African Journal of Psychology, 35(4), 766-792.

Twohig, M. P., \& Crosby, J. M. (2010). Acceptance and commitment therapy as a treatment for problematic Internet pornography viewing. Behavior Therapy, 41, 285295.

Thombs, D., \& Osborn, C. (2001). A cluster analytic study of clinical orientations among chemical dependency counsellors. Journal of Counseling and Development, 79 , 450-457
Toriello, P., \& Leierer, S. (2005). The relationship between clinical orientation of substance abuse professionals and their clinical decisions. Rehabilitation Counseling Bulletin, $48,75-88$.

Warden, N. L., Phillips, J. G., \& Ogloff, J. R. P. (2004). Internet Addiction. Psychiatry, Psychology and Law, 11(2), $280-295$.

Weinstein, A. \& Lejoyeax, M. (2010). Internet Addiction or Excessive Internet Use. The American Journal of Drug and Alcohol Abuse, 36, 277-283.

Westland, S., Shinebourne, P. (2009). Self-deception and the therapist: An interpretative phenomenological analysis of the experiences and understandings of therapists working with clients they describe as self-deceptive. Psychology and Psychotherapy: Theory, Research and Practice, 82, 385-401.

Widyanto, L., \& Griffiths, M. (2006). Internet addiction: A critical review. International Journal of Mental Health and Addiction, 4(1), 31-51.

Widyanto, L., \& McMurren, M. (2004). The psychometric properties of the Internet Addiction Test. CyberPsychology and Behaviour (1), 237-244.

Young, K. S. (1996). Psychology of computer use: Addictive use of the Internet: A case that breaks the stereotype. Psychological Reports., 79, 899-902.

Young, K. (1999). Internet addiction: Evaluation and treatment. Student British Medical Journal. 7, 351-352.

Young, K. S. (2007). Cognitive-behavioural therapy with Internet addicts: Treatment outcomes and implications. CyberPyschology \& Behaviour, 10(5), 671-679.

Young, K. S., \& Abreu, C. N. (2011). Internet Addiction - A Handbook and Guide to Evaluation and Treatment New Jersey: John Wiley \& Sons.

Young, K., Pistner, M., O'Mara, J., \& Buchanan, J. (2000). Cyber-disorders: The mental health concern for the new millennium. CyberPsychology and Behavior, 3, 475-479.

Young, K. S., Yue, X. D., \& Ying, L. (2011). Prevalence estimates and etiologic models of Internet addiction. In K. S. Young \& C. N. Abreu (Eds.), Internet Addiction - $A$ Handbook and Guide to Evaluation and Treatment. New Jersey: John Wiley and Sons.

Zeigarnik, B. V. (1967). On finished and unfinished tasks. In W. D. Ellis (Ed.). A source book of Gestalt psychology., (p. 300-315). New York: Humanities Press. 